\title{
Perforación esofágica cervical tardía por rotura y desplazamiento de placa de artrodesis cervical anterior
}

\author{
Jaime Ruiz-Tovar, Montiel Jiménez-Fuertes, Gustavo Díaz, \\ Juan González, César Levano-Linares y Manuel Durán
}

Servicio de Cirugía General y del Aparato Digestivo. Hospital Universitario Rey Juan Carlos. Madrid, España.

Recibido el 25 de junio de 2017, aceptado para publicación el 30 de agosto de 2017 .

Correspondencia a: Jaime Ruiz-Tovar jruiztovar@gmail.com

\section{Delayed cervical esophageal perforation by rupture and displacement} of anterior cervical arthrodesis plaque

Introduction: Esophageal perforation is a possible complication after anterior cervical fusion. However, these complications usually appear intraoperatively or in the early postoperative course. Case report: A 35-years-old females, who underwent a C3-C5 anterior cervical fusion 3 years ago, after suffering a mild cervical trauma, she complained of dysphagia. A retroesophageal abscess was observed, caused by esophageal perforation, secondary to plaque rupture and screw extrusion.

Key words: esophageal perforation; anterior cervical fusion; retroesophageal abscess.

\section{Resumen}

Introducción: La perforación esofágica es una posible complicación de la artrodesis cervical anterior. Sin embargo, estas suelen ocurrir intraoperatoriamente o en el posoperatorio precoz. Caso clínico: Mujer de 35 años sometida, 3 años antes, a artrodesis de C3-C5, que tras sufrir un traumatismo leve con latigazo cervical, comienza con disfagia. Se objetiva un absceso retroesofágico por perforación esofágica, causado por rotura de la placa protésica y extrusión de un tornillo.

Palabras clave: perforación esofágica; artrodesis cervical anterior; absceso retroesofágico.

\section{Introducción}

La artrodesis cervical anterior es una intervención realizada con mucha frecuencia para el tratamiento de mielopatías o radiculopatías cervicales o en casos de lesiones espinales cervicales. Aunque suele ser un procedimiento seguro, pueden aparecer complicaciones, entre las que se incluyen lesiones medulares o radiculares, del nervio laríngeo recurrente y de grandes vasos cervicales. La lesión esofágica es una complicación muy poco frecuente, con una incidencia estimada entre 0,02 y $1,5 \%$, pero con una mortalidad que puede alcanzar hasta el $20 \%{ }^{1-3}$. La lesión esofágica se puede clasificar en intraoperatoria, posoperatoria precoz (durante el primer mes posoperatorio) o posoperatoria tardía (apareciendo meses después de la cirugía). Las dos primeras están ampliamente descritas en la literatura. Sin embargo, la perforación esofágica que ocurre meses o inclu- so años después de la intervención, es un hecho extremadamente raro, existiendo muy pocos casos descritos en la literatura.

\section{Caso clínico}

Mujer de 35 años, embarazada de 21 semanas, con antecedente de fractura de cuerpos vertebrales C5 y C7 por accidente de tráfico 3 años antes y habiéndose realizado una artrodesis de 2 espacios con caja intersomática, acude al Servicio de Urgencias por disfagia de $24 \mathrm{~h}$ de evolución y sensación febril. La paciente refería haber tenido un accidente de tráfico de baja energía un mes antes, aunque con latigazo cervical, pero sin ninguna sintomatología en ese momento. A la exploración física no presentaba dolor, fluctuación ni enfisema cervical.

Una analítica sanguínea mostró 12.000 leuco- 
citos $/ \mathrm{mm}^{3}$ y una proteína c reactiva de $4,02 \mathrm{mg} / \mathrm{dl}$, como únicos hallazgos significativos. La paciente fue valorada por otorrinolaringología mediante fibroscopia, sin hallazgos significativos.

Se realizó una resonancia magnética cervical, que evidenció un desplazamiento anterior del tornillo de fijación de $\mathrm{C} 5$, que se encuentra rodeado de una colección líquida de 25 × 35 × 41 mm de diámetros, con nivel hidroaéreo, sugestivo de absceso en el espacio retroesofágico. Además, se apreciaba una hiperintensidad de señal esofágica a ese nivel, sugestiva de posible perforación esofágica como etiología del absceso (Figura 1).

Ante estos hallazgos, se decidió realizar una cirugía urgente. A través de una incisión cervical anterior con ampliación lateral izquierda, se incide hasta el espacio prevertebral, donde se localiza el absceso de contenido purulento. Se aprecia placa de artrodesis rota a nivel de la arandela de C5 con el tornillo de fijación de ese nivel parcialmente extruido (Figura 2). Se decide retirar todos los tornillos de fijación y la placa, evidenciándose la caja intersomática C5-C6 normoposicionada sin movimiento aparente a la tracción. Se moviliza el esófago parcialmente hasta poder visualizar su cara posterior, observándose una perforación de $0,5 \mathrm{~cm}$ a ese nivel (Figura 3). Se sutura la perforación con 2 puntos de poliglactina 910 (Vicryl $^{\circledR}$, Johnson \& Johnson, USA) de $2 / 0$ y se dejan 2 drenajes tipo penrose en la cavidad del absceso y el espacio retroesofágico. Se pauta: tratamiento antibiótico con piperacilina/tazobactam 4,5 g cada 8 h y nutrición parenteral total.

La paciente evolucionó favorablemente. Se realizó un esofagograma de control al $7^{\circ}$ día posoperatorio sin evidencia de fuga de contraste, por lo que se inició la alimentación por vía oral, siendo dada de alta la paciente al $10^{\circ}$ día posoperatorio.

A los 6 meses de la intervención, la paciente está asintomática, tanto desde el punto de vista digestivo como traumatológico.

\section{Discusión}

Newhouse y cols analizaron los datos de 10.000 pacientes operados de artrodesis cervical anterior e incluidos en la base de datos de la cervical Spine Research Society, encontrando 22 casos de peroforación esofágica, de los cuales 6 se detectaron intraoperatoriamente, 6 durante el posoperatorio inmediato y 10 casos fueron tardíos, diagnosticándose semanas después de la intervención ${ }^{4}$. Gaudinez y cols ${ }^{5}$ identificaron 44 perforaciones esofágicas en una serie de 2.946 pacientes, de las cuales el $77 \%$

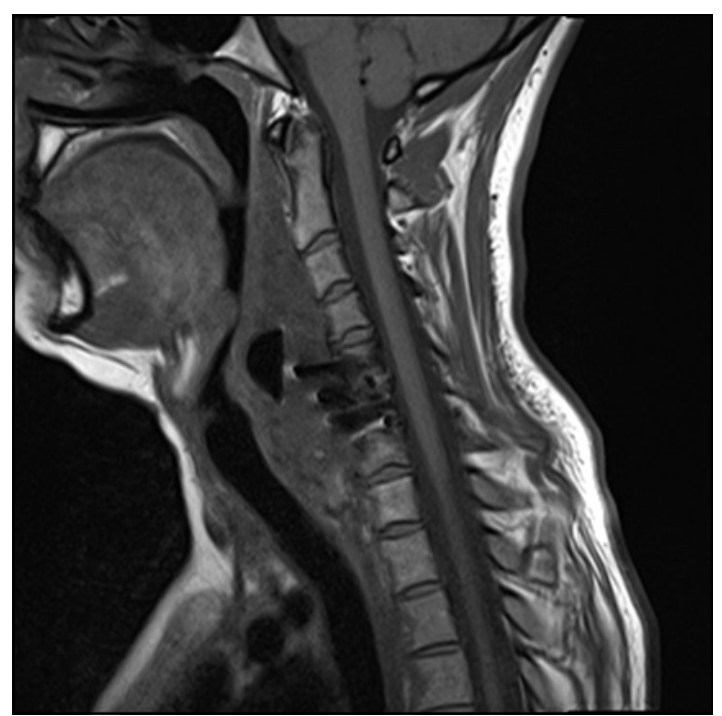

Figura 1. RM. Corte sagital. Imagen potenciada en T1. Extrusión de tornillo de fijación de la placa en C5 y contacto con absceso retroesofágico con nivel hidroaéreo.

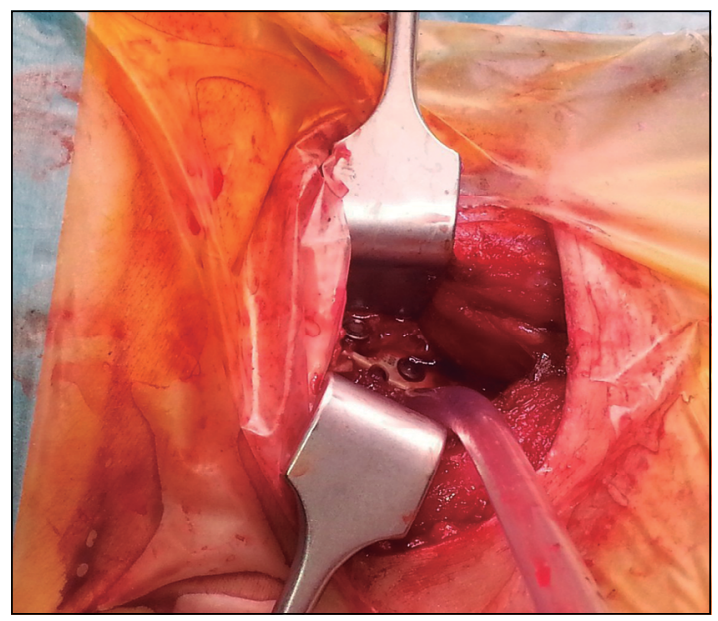

Figura 2. Extrusión de tornillo y rotura de la placa protésica.

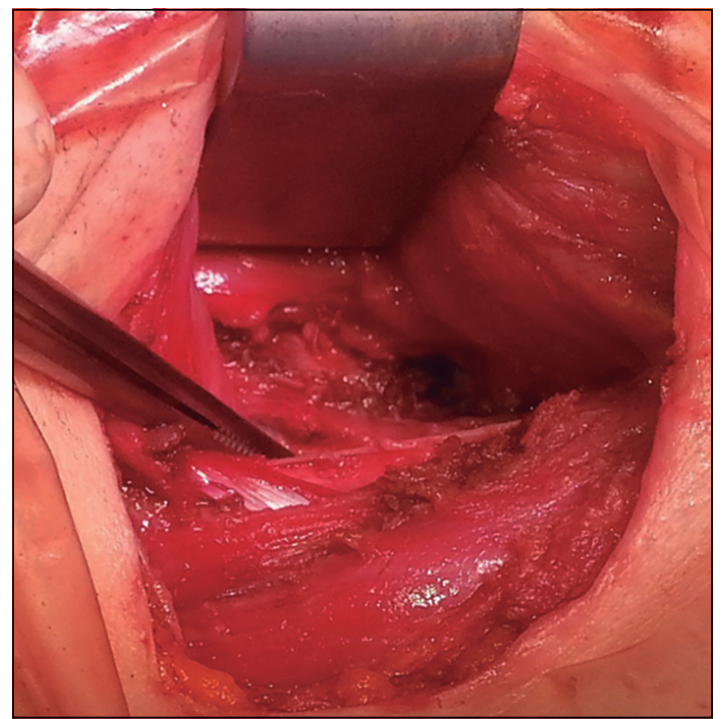

Figura 3. Perforación esofágica (señalada por la pinza) 
ocurrieron intraoperatoriamente y el resto durante el posoperatorio inmediato o semanas o meses tras la intervención. La aparición de la perforación esofágica años tras la intervención, como ocurrió en nuestra paciente, es extremadamente rara, aunque hay casos descritos en la literatura, tal y como describen Yang y cols, que observaron una perforación esofágica 8 años tras la artrodesis ${ }^{2}$. Acosta-Mérida y cols describieron recientemente un caso de perforación esofágica en nuestreo país, un año tras haber sido sometida a la artrodesis ${ }^{6}$.

Las causas descritas de las perforaciones tardías son extrusión del implante, compresión del material protésico sobre el esófago o fricción crónica del implante sobre la pared posterior del esófago durante la deglución, condicionando una necrosis por presión ${ }^{7,8}$. En nuestra paciente, la causa de la perforación esofágica fue la rotura de la arandela para inserción de uno de los tornillos en la placa, condicionando la extrusión del tornillo. Esta rotura de la placa posiblemente se produjo durante un traumatismo de baja energía, pero con latigazo cervical, que ocurrió unas semanas antes. En ese momento se produciría la rotura de la placa y lentamente se iría soltando el tornillo hasta alcanzar la cara posterior del esófago y erosionarlo progresivamente hasta producir una perforación. Hasta la fecha, este es el primer caso descrito en la literatura de perforación esofágica por este mecanismo causal.

El síntoma principal en las perforaciones tardías es la disfagia, que ocurre en más del $50 \%$ de los casos descritos. También puede manifestarse como tos, picos febriles aislados o enfisema subcutáneo. La presencia de estos síntomas en un paciente con antecedente de artrodesis cervical anterior debe hacer sospechar una posible perforación esofágica ${ }^{5}$. La infección puede extenderse a tejidos vecinos, originando una mediastinitis, erosión de grandes vasos en el cuello o abscesos cervicales, aumentando el riesgo de mortalidad asociada al proceso ${ }^{2,8}$.

Aunque la extrusión o rotura de los implantes protésicos pueden diagnosticarse mediante radiografía cervical simple, las técnicas de imagen de elección para el diagnóstico de perforación esofágica son la TC cervical con contraste oral o la RM, con una sensibilidad diagnóstica del $80 \%{ }^{9}$. En nuestro caso se optó por realizar una RM, ante el embarazo de la paciente. La esofagoscopia también puede ser de utilidad para evaluar el tamaño de la perforación esofágica.

El tratamiento básico de las perforaciones esofágicas tardías es el drenaje del absceso, la retirada del material protésico y la reparación de la perforación. El tratamiento debe instaurarse de forma precoz para evitar la progresión a complicaciones fatales, como puede ser la mediastinitis. Se han descrito casos de cierre espontáneo de la perforación con tratamiento conservador (antibioterapia) en perforaciones menores de $1 \mathrm{~cm}$. Sin embargo, la cirugía es el tratamiento de elección en perforaciones de mayor tamaño o cuando hay signos de infección local. El cierre de la perforación puede realizarse con o sin interposición de colgajo muscular, siendo el flap más frecuentemente empleado el de esternocleidomastoideo $^{10}$. Los colgajos musculares suelen ser necesarios en perforaciones tardías de gran tamaño, con el fin de establecer una barrera entre el esófago y la columna vertebral, pero además, permiten una correcta vascularización para la cicatrización de la perforación. En nuestro caso, a pesar de que la perforación era tardía, el pequeño tamaño de la misma permitió que la sutura simple fuera suficiente para cerrar el orificio sin tensión, permitiendo una correcta vascularización.

\section{Conclusión}

La perforación esofágica tardía tras artrodesis cervical anterior es muy poco frecuente. Sin embargo, debe tenerse en cuenta en pacientes con disfagia. Un antecedente reciente de traumatismo cervical debe hacer sospechar una posible rotura del material protésico como causa etiológica. El tratamiento debe realizarse de forma precoz para evitar la progresión a mediastinitis, erosión de grandes vasos o sepsis.

\section{Responsabilidades éticas}

Protección de personas y animales. Los autores declaran que para esta investigación no se han realizado experimentos en seres humanos ni en animales.

Confidencialidad de los datos. Los autores declaran que han seguido los protocolos de su centro de trabajo sobre la publicación de datos de pacientes.

Derecho a la privacidad y consentimiento informado. Los autores declaran que en este artículo no aparecen datos de pacientes.

Conflicto de intereses: Los autores declaran no tener conflicto de intereses en la publicación de este artículo. 


\section{Referencias}

1. Krishnan P, Kartikueyan R, Patel SM, et al. Two cases of esophageal injury following anterior cervical discectomy and fusión: one overt and one covert. Ann Neurosci 2017;24:126-30.

2. Yang S, Lee SB, Cho Ks. Delayed esophagus perforation after anterior cervical spine surgery. Korean $\mathrm{J}$ Neurotrauma 2015;11:191-4

3. Amhaz HH, Kuo R, Vaidya R, Orlewicz MS. Esopahgeal perforation following cervical spine surgery: a review with considerations in airway management. Int J Crit Illn Inj Sci 2013;3:276-8.

4. Newhouse KE, Lindsey RW, Clark CR, Lieponis J, Murphy MJ. Esophageal perforation following anterior cervical spine surgery. Spine 1989;14:1051-3.

5. Gaudinez RF, English GM, Gebhard JS, Brugman JL, Donaldson DH, Brown CW. Esopahgeal perforations after anterior cervical surgery. J Spinal Disord 2000;13:77-84.

6. Acosta-Mérida MA, Medina-Velázquez R, Marchena-Gómez J, Alonso-Gómez J, Mhaildli H. Disfagia tras artrodesis cervical por migración del material protésico. Cir Esp 2015;93:537-9.

7. Witwer BP, Resnick DK. Delayed esophageal injury without instrumentation failure: complication of anterior cervical instrumentation. J Spinal Disord Tech 2003;16:519-23.

8. Zdichavsky M, Blauth M, Bosch U,
Rosenthal H, Knop C, Bastián L. Late esophageal perforation complicating anterior cervical plate fixation in ankylosing spondylitis: a case report and review of the literature. Arch Orthop Trauma Surg. 2004;124:349-53.

9. Sharma RR, Sethu AU, Lad SD, Turel KE, Pawar SJ. Pharyngeal perforation and spontaneous extrusion of the cervical graft with its fixation device: a late complication of $\mathrm{C} 2-\mathrm{C} 3$ fusion via anterior approach. J Clin Neurosci. 2001;8:464-8.

10. Navarro R, Javahery R, Eismont F, Arnold DJ, Bhatia NN, Vanni S, et al. The role of the sternocleidomastoid muscle flap for esophageal fistula repair in anterior cervical spine surgery. Spine 2005;30:E617-22. 